Silvia Cypriano 1

Giovana Daniela Pecharki 1

Maria da Luz Rosário de Sousa 1

Ronaldo Seichi Wada 1

\section{A saúde bucal de escolares residentes em locais com ou sem fluoretação nas águas de abastecimento público na região de Sorocaba, São Paulo, Brasil}

\author{
Oral health of schoolchildren residing in areas \\ with or without water fluoridation in Sorocaba, \\ São Paulo State, Brazil
}

1 Faculdade de Odontologia de Piracicaba, Universidade Estadual de Campinas. Av. Limeira 901, Piracicaba, SP 13414-018, Brasil. silviacypriano@hotmail.com g_pecharki@yahoo.com.br luzsousa@fop.unicamp.br rwada@fop.unicamp.br

\begin{abstract}
This study describes dental caries data and fluorosis in seven municipalities in the Sorocaba region, São Paulo State, Brazil, comparing those with fluoridated as opposed to nonfluoridated water. Criteria were those proposed by the World Health Organization (WHO, 1997), adapted by the School of Public Health, University of São Paulo. The sample was selected at random, without replacement, and was calculated by age, accepting a design error of 2 and a sampling loss of 20\% ( $n=96$ per age). There were six trained examiners, with accepted agreement percentage above $89 \%$ for all variables. In the Sorocaba region, the dmft index in 5 year-old children was 3.1, and 37.6\% were caries-free $(D M F T=0)$. At age 12, 32.3\% were caries-free $(D M F T=$ 0 ), and the DMFT index in the region was 2.6, thus characterizing the area as having a low prevalence of caries. In areas with fluoridated water, the proportion of caries-free children at 12 years was higher than in those without fluoridated water $(p=0.019)$, and the DMFT was lower $(p=0.001)$. Prevalence of fluorosis at age 12 was $12.7 \%$ in the Sorocaba area and did not pose a public health problem according to the community fluorosis index (CFI).
\end{abstract}

Key words Dental Caries; Fluoridation; Dental Fluorosis; Child Health; Oral Health

Resumo Este estudo apresenta dados de cárie dentária e fluorose em sete municípios representativos da região de Sorocaba, São Paulo, Brasil, que possuem ou não flúor nas águas de abastecimento público. A metodologia utilizada foi proposta pela Organização Mundial da Saúde (WHO, 1997), adaptada pela Faculdade de Saúde Pública da Universidade de São Paulo. A amostra foi probabilística, admitindo um erro de desenho de 2 e perda amostral de 20,0\% ( $n=$ 96 por idade). Foram calibrados seis examinadores, com porcentagem de concordância acima de 89\% para as variáveis pesquisadas. Obteve-se o resultado de 37,6\% de crianças livres de cárie aos 5 anos $(c e o d=0)$ e o indice ceod foi de 3,1. Aos 12 anos, 32,3\% dos escolares tinham CPOD 0 e o índice CPOD foi de 2,6, sendo a região considerada de baixa prevalência de cárie. Nos municípios com água fluoretada a proporção de crianças livres de cárie aos 12 anos foi maior ( $p=$ $0,019)$, e o índice ceod foi menor ( $p=0,001$ ). A prevalência de fluorose nas crianças examinadas foi de 12,7\%, estando dentro de limites aceitáveis segundo o índice de fluorose dentária da comunidade.

Palavras-chave Cárie Dentária; Fluoração; Fluorose Dentária; Saúde Infantil; Saúde Bucal 


\section{Introdução}

Em grande parte dos países desenvolvidos (Marthaler et al., 1996), inclusive no Brasil (Bronecker et al., 2002; Cury, 2001; Dini et al., 1999; Traebert et al., 2001), tem sido observado um declínio na prevalência de cárie dental. As causas mais prováveis para essa queda estão associadas ao uso generalizado de fluoretos, acesso aos serviços odontológicos, mudanças de critérios de diagnóstico de cárie e ampliação das ações de promoção e educação em saúde bucal (Nadanovsky, 2000; Nadanovsky \& Sheiham, 1995). Paralelamente a esse declínio de cárie, tem sido relatado um aumento na prevalência de fluorose em várias localidades do mundo nas últimas décadas (Beltran-Aguilar et al., 2002; Fomon et al., 2000; Jackson et al., 1999).

Sendo assim, é fundamental o conhecimento da distribuição e tendência de evolução dessas doenças no território brasileiro, pois a cárie dental certamente ainda representa um problema de saúde pública, e a fluorose dentária é uma condição que deve ser permanentemente monitorada. Dessa forma, conforme preconiza a Lei 8.080 de 1990 (Brasil, 1990), a epidemiologia deveria ser utilizada nesse processo a fim de que medidas apropriadas para redução e controle das doenças fossem implementadas.

Além disso, a Organização Mundial da Saúde - OMS - (WHO, 1987) estabelece que esses estudos sejam realizados a cada cinco anos, permitindo, assim, um adequado acompanhamento do estado de saúde bucal da população e avaliação das ações desenvolvidas.

No Brasil, estudos realizados em 1986 e 1996 pelo Ministério da Saúde (MS), somente nas capitais, demonstraram uma diminuição na prevalência de cárie, permanecendo dúvidas se os dados obtidos são aplicáveis para cidades do interior (Narvai \& Castellanos, 1999; Traebert et al., 2001). Com o objetivo de melhorar o conhecimento da distribuição e tendência de algumas doenças bucais, a Secretaria do Estado de Saúde de São Paulo (SES/SP) realizou, no ano de 1998, um amplo estudo epidemiológico, envolvendo 133 municípios paulistas (Narvai \& Castellanos, 1999). A região de Sorocaba (Direção Regional de Saúde - DIR XXIII), com sete municípios sorteados, também participou da amostra estadual.

Observa-se, porém, que as informações epidemiológicas, obtidas em nível municipal, são pouco utilizadas e, muitas vezes, sequer chegam a ser publicadas. Com isso, esses estudos são subutilizados, e seus achados e conclusões raramente geram soluções efetivas, ficando inexplorado o potencial dos dados produzidos (Traebert et al., 2002).

Desse modo, o objetivo deste estudo foi verificar e divulgar a prevalência da cárie dentária e fluorose em pré-escolares e escolares na região de Sorocaba, acrescentando informações relativas a estas variáveis de crianças residentes em locais com ou sem água de abastecimento público fluoretada.

\section{Material e métodos}

A pesquisa multicêntrica, sob a coordenação científica da Faculdade de Saúde Pública da Universidade de São Paulo (FSP/USP) e SES/SP, abrangeu as 24 Direções Regionais de Saúde do Estado de São Paulo (DIR), e, para a região de Sorocaba (DIR XXIII), sete municípios, dentre os 48 existentes, foram sorteados para compor a amostra da região, de acordo com os seguintes critérios: porte do município (os municípios grandes eram os que continham acima de 50 mil habitantes; os de pequeno porte os que tinham até 10 mil habitantes; e os de médio porte entre esses valores) e a presença ou não de água fluoretada, segundo as informações oficiais obtidas na ocasião (Narvai \& Castellanos, 1999). Foram sorteados um município de porte grande (Itapetininga), dois municípios de porte médio (Iperó com água fluoretada e Porto Feliz sem água fluoretada) e quatro municípios de porte pequeno (sendo dois com água fluoretada - Itaoca e Barra do Chapéu, e dois sem água fluoretada - Bom Sucesso do Itararé e Itapirapuã Paulista).

O Município de Porto Feliz, na ocasião do sorteio, foi incluído no grupo de municípios que não tinham fluoretação das águas de abastecimento público. Entretanto, conforme informações da Secretaria Municipal de Saúde, esse município tem suas águas fluoretadas desde 1981. Foram, então, coletadas amostras de água de todas as escolas do município, a fim de verificar os teores do íon flúor. A média encontrada foi de 0,53ppm de flúor (mínimo de 0,47ppm e máximo de 0,59ppm de flúor), segundo o Laboratório de Bioquímica da Faculdade de Odontologia (FOP) da Universidade Estadual de Campinas (UNICAMP). Dessa forma, neste estudo, Porto Feliz foi incluído no grupo de municípios com fluoretação das águas de abastecimento público, para possibilitar uma análise mais próxima à realidade.

O tamanho amostral por idade e por município foi calculado consoante recomendação da OMS (WHO, 1987), em que se obtiveram 96 elementos amostrais para cada idade ou faixa 
etária. Esse valor foi obtido mediante a consideração de que quarenta crianças por idade são suficientes para obtenção de dados confiáveis, quando a prevalência da cárie é moderada ou alta. Optou-se por admitir um erro de desenho de 2 e também a perda amostral de $20 \%$, daí 40 $\mathrm{x} 2+20 \%=96$ (Frias, 2000).

Os espaços escolares, públicos e particulares, de cada município foram identificados com base no cadastro fornecido pela Secretaria de Estado da Educação. No caso de municípios com mais de vinte escolas de cada tipo, realizou-se o sorteio de vinte pré-escolas, vinte escolas de ensino fundamental e vinte de ensino médio, definindo-se, dessa maneira, as unidades amostrais de cada município, para, a partir daí, obter a listagem dos alunos. Nos municípios com até vinte escolas, incluíram-se todos os espaços escolares para o sorteio das crianças.

Após a aprovação do Comitê de Ética em Pesquisa da FSP/USP (Processo no 62/98), obteve-se a autorização dos responsáveis pelas crianças para a realização dos exames, pelo termo de consentimento livre e esclarecido, com as devidas informações do que estaria sendo realizado.

Os elementos amostrais foram selecionados valendo-se de sorteio e constituídos de pré-escolares de 5 e 6 anos e escolares de 7 a 12 anos, e indivíduos de outros grupos etários que não serão apresentados neste estudo.

A coordenação científica da região de Sorocaba foi realizada pela FOP/UNICAMP. Os índices que foram utilizados obedeceram aos códigos e critérios recomendados pela OMS (WHO, 1997). Foram pesquisadas as seguintes condições: cárie dentária (índices CPOD e ceod) e a fluorose dentária (índice de Dean), além de outras condições de saúde bucal, que no entanto não serão relatadas no presente estudo.

A calibração foi realizada no município de Sorocaba, totalizando quarenta horas divididas entre discussões teóricas e atividades práticas, simulando as diferentes condições e situações que os examinadores encontrariam durante a realização do trabalho prático.

Em relação à técnica adotada, seguiu-se a recomendação da coordenação geral da pesquisa: a técnica do consenso (Narvai \& Castellanos, 1999). Os registros relativos aos dois últimos dias foram considerados para o cálculo do erro interexaminadores.

A porcentagem de discordância interexaminadores foi de: $1,8 \%$ para a coroa, $1,1 \%$ para a necessidade de tratamento e $10,6 \%$ para a fluorose. Esses resultados são aceitáveis para estu- dos exploratórios (WHO, 1987), comprovando a validade dos dados coletados (Frias, 2000).

A coleta de dados foi realizada após a calibração por seis examinadores, em local com luz natural, sendo bem iluminado e ventilado e próximo à fonte de água, com o examinador sentado em 12 horas em relação à cabeça da criança, que se manteve deitada. Para os exames, utilizou-se espelho bucal plano número 5 e a sonda CPI ("ball-point") para levantamentos epidemiológicos. Os registros foram realizados em fichas individuais propostas pela FSP/USP (Narvai \& Castellanos, 1999).

Os dados foram digitados na FSP/USP, utilizando para tal o programa Epi-Info versão 5.01 e o programa EPIBUCO para o processamento e análise dos dados (Narvai \& Castellanos, 1999).

Utilizou-se também o Índice de Fluorose Dentária da Comunidade (IFC), que é calculado atribuindo-se uma nota ou grau a cada criança de acordo com o índice de Dean. Calcula-se então a média aritmética das notas atribuídas a cada criança, de acordo com a fórmula: $\sum$ (freqüência $x$ grau)/n, onde " $n$ " corresponde ao número de crianças examinadas (Chaves, 1977). Com base no valor obtido, verificase o seu significado do ponto de vista da saúde pública.

Para a análise estatística dos dados, utilizou-se o teste qui-quadrado para verificar se houve diferenças entre os grupos analisados, com nível de significância de 5,0\% e o teste " $\mathrm{t}$ " para comparação de duas médias, para verificar se houve diferenças nos valores médios dos índices ceod e CPOD. Tais procedimentos, a despeito das discrepâncias observadas entre os tamanhos de amostras, têm um caráter exploratório e deverão fornecer subsídios que permitam a realização de estudos posteriores mais acurados.

\section{Resultados}

Este estudo apresenta os dados de prevalência e severidade de cárie de sete municípios representativos da região de Sorocaba. Examinaram-se 3.731 crianças de 5 a 12 anos (Tabela 1).

Na dentição decídua, o índice ceod aumenta proporcionalmente de 3,1 a 3,8 até a idade de oito anos e depois diminui, até atingir 0,3 aos 12 anos (Tabela 2). Em relação aos componentes do índice ceod, verifica-se um predomínio de dentes cariados em todas as idades. Nas crianças de 5 a 12 anos, a média do índice ceod foi estatisticamente menor nos municípios com fluoretação. Nas idades de 5, 6, 8 e 12 anos, 
constataram-se diferenças estatisticamente significantes.

Na dentição permanente, o índice CPOD aumenta proporcionalmente com a idade, apresentando aos 12 anos um CPOD médio de 2,6 (Tabela 3). Considerando os componentes do índice CPOD, verificou-se que a proporção de dentes cariados foi maior em todas as idades, e a menor porcentagem foi observada aos 12 anos $(57,8 \%)$. Não houve diferenças estatisticamente significativas entre as médias do índice CPOD das crianças de 5 a 12 anos residentes em áreas fluoretadas e não fluoretadas, exceto aos seis anos de idade.

Tabela 1

Número de indivíduos examinados, considerando o local de residência, segundo a presença ou não de flúor na água de abastecimento público e a faixa etária. Região de Sorocaba, São Paulo, Brasil, 1998.

\begin{tabular}{|c|c|c|c|c|c|c|}
\hline \multirow[t]{3}{*}{ Idade } & \multicolumn{4}{|c|}{ Residência } & \multirow{2}{*}{\multicolumn{2}{|c|}{ Total }} \\
\hline & \multicolumn{2}{|c|}{ Área fluoretada } & \multicolumn{2}{|c|}{ Área não fluoretada } & & \\
\hline & $\mathrm{n}$ & $\%$ & $\mathrm{n}$ & $\%$ & $n$ & $\%$ \\
\hline 5 & 337 & 89,8 & 38 & 10,2 & 375 & 100,0 \\
\hline 6 & 348 & 87,2 & 51 & 12,8 & 399 & 100,0 \\
\hline 7 & 390 & 85,7 & 65 & 14,3 & 455 & 100,0 \\
\hline 8 & 415 & 83,3 & 83 & 16,7 & 498 & 100,0 \\
\hline 9 & 438 & 83,3 & 88 & 16,7 & 526 & 100,0 \\
\hline 10 & 417 & 82,5 & 85 & 17,5 & 502 & 100,0 \\
\hline 11 & 453 & 85,9 & 74 & 14,1 & 527 & 100,0 \\
\hline 12 & 393 & 87,5 & 56 & 12,5 & 449 & 100,0 \\
\hline Total & 3.191 & 85,5 & 540 & 14,5 & 3.731 & 100,0 \\
\hline
\end{tabular}

O índice CPOD aos 12 anos no município de grande porte com flúor (Itapetininga) foi de 1,9; nos municípios de médio porte com flúor foi de 2,2 (Porto Feliz) e 2,7 (Iperó); nos municípios de pequeno porte com flúor (Itaoca e Barra do Chapéu) foi, respectivamente, de 2,3 e 3,4 , e nos de pequeno porte e sem fluoretação foi de 2,6 (Bom Sucesso do Itararé) e 3,2 (Itapirapuã Paulista).

Levando-se em conta a distribuição da freqüência do índice CPOD aos 12 anos, 32,3\% dos escolares apresentaram um CPOD igual a zero $(n=145), 37,6 \%(n=169)$ apresentaram CPOD entre 0 e 3 , e $30,1 \%(n=135)$, um CPOD maior que 3 .

O percentual de crianças livres de cárie aos cinco anos $(\operatorname{ceod}=0)$ foi de $37,3 \%$.

Verificou-se em todas as idades maior porcentagem de crianças livres de cárie nos municípios com fluoretação das águas de abastecimento público. O teste qui-quadrado mostrou haver diferença estatisticamente significativa aos 5, 6, 8, 9 e 12 anos de idade (Figura 1).

Tomando-se por base o grupo etário de 7 a 12 anos $(n=2.897)$, a prevalência de fluorose dentária foi de $12,9 \%$, sendo menor nas crianças que residiam nos municípios com flúor nas águas de abastecimento público do que nas crianças que residiam nos municípios sem esse benefício, diferindo estatisticamente aos 7, 10 e 11 anos, e apresentando semelhanças nas demais idades (Tabela 4). Quanto aos graus de fluorose, $87,3 \%$ das crianças foram incluídas nas categorias normal ou questionável, $8,2 \%$ muito leve, $2,2 \%$ leve, $0,8 \%$ moderada e $0,1 \%$ na categoria severa.

Tabela 2

Média de dentes erupcionados, porcentagem de dentes cariados, extraídos e obturados e índice ceod,

segundo a área de residência e a idade. Região de Sorocaba, São Paulo, Brasil, 1998.

\begin{tabular}{|c|c|c|c|c|c|c|c|c|c|c|}
\hline \multirow[t]{3}{*}{ Idade } & \multirow{3}{*}{$\begin{array}{l}\text { Erupcionados } \\
\text { Média }\end{array}$} & \multirow{3}{*}{$\begin{array}{l}\text { ceod } \\
\text { Média }\end{array}$} & \multirow{3}{*}{$\begin{array}{c}\text { Cariados } \\
(\%)\end{array}$} & \multirow{3}{*}{$\begin{array}{c}\text { Extraídos } \\
(\%)\end{array}$} & \multirow{3}{*}{$\begin{array}{l}\text { Obturados } \\
(\%)\end{array}$} & \multicolumn{4}{|c|}{ ceod } & \multirow{3}{*}{$\begin{array}{c}\text { Nível de } \\
\text { significância } \\
P\end{array}$} \\
\hline & & & & & & \multicolumn{2}{|c|}{ Área com fluoretação } & \multicolumn{2}{|c|}{ Área sem fluoretação } & \\
\hline & & & & & & Média & IC95\% & Média & IC95\% & \\
\hline 5 & 18,5 & 3,1 & 83,7 & 0,8 & 15,5 & $2,8 a$ & $(2,4-3,1)$ & $5,5 b$ & $(4,0-7,1)$ & $p<0,0001$ \\
\hline 6 & 16,3 & 3,3 & 83,8 & 0,5 & 15,7 & $3,2^{a}$ & $(2,8-3,5)$ & $4,3 b$ & $(3,2-5,3)$ & $p=0,035$ \\
\hline 7 & 13,2 & 3,8 & 82,0 & 1,1 & 16,9 & $3,6 a$ & $(3,3-4,0)$ & $4,5 \mathrm{a}$ & $(3,6-5,4)$ & $p=0,051$ \\
\hline 8 & 10,8 & 3,3 & 79,9 & 1,7 & 18,4 & $3,1 \mathrm{a}$ & $(2,9-3,4)$ & $4,2 b$ & $(3,6-4,9)$ & $p=0,002$ \\
\hline 9 & 8,1 & 2,5 & 78,5 & 1,2 & 20,3 & $2,5 a$ & $(2,2-2,7)$ & $2,8 a$ & $(2,3-3,3)$ & $p=0,216$ \\
\hline 10 & 4,6 & 1,4 & 69,6 & 1,0 & 29,5 & $1,4 a$ & $(1,2-1,6)$ & $1,4 a$ & $(1,0-1,7)$ & $p=0,751$ \\
\hline 11 & 1,8 & 0,6 & 65,3 & 0,7 & 34,1 & $0,6 a$ & $(0,5-0,7)$ & $0,6 a$ & $(0,4-0,9)$ & $p=0,798$ \\
\hline 12 & 0,7 & 0,3 & 63,6 & 0,0 & 36,4 & $0,2^{a}$ & $(0,1-0,3)$ & $0,7 b$ & $(0,6-0,8)$ & $p<0,0001$ \\
\hline 5 a 12 & 8,7 & 2,2 & 79,6 & 1,0 & 19,4 & $2,1 \mathrm{a}$ & $(2,0-2,2)$ & $2,8 b$ & $(2,5-3,1)$ & $p<0,0001$ \\
\hline
\end{tabular}

Nota: Letras seguidas de números na horizontal diferem entre si no nível de significância de $5 \%$. 
Aplicou-se o índice de fluorose dentária da comunidade cujo valor foi de 0,19 e, segundo Russell, esse nível de fluorose não é considerado um problema de saúde pública, mas de alto valor sob o ponto de vista de prevenção da cárie dentária (Chaves, 1977).

\section{Discussão}

Uma das limitações deste estudo se refere à pré-estratificação dos municípios das regiões do Estado de São Paulo, segundo o porte do município e ter ou não água fluoretada em número igual para todas as regiões, o que pode não corresponder proporcionalmente à realidade, restringindo a inferência para algumas regiões do Estado de São Paulo e para a região estudada. Em acréscimo, a realização de testes estatísticos por idade, apesar das grandes diferenças observadas entre tamanhos de amostra, permitiram que se obtivessem informações sobre as variáveis em estudo, assim como, de forma exploratória permitiram obter elementos que possibilitarão a elaboração e o desenvolvimento de novos estudos, conduzidos de maneira mais acurada.

Este estudo identifica a atual situação de saúde bucal de sete municípios representativos de uma importante região do Estado de São Paulo, acrescentando informações sobre a prevalência de cárie e fluorose, segundo o local de residência, considerando a presença ou ausência de flúor das águas de abastecimento público, úteis para o planejamento regional em saúde bucal e subsídio dos serviços locais.
Os resultados foram apresentados no conjunto de municípios para possibilitar as análises estatísticas. Apenas aos 12 anos o índice CPOD também foi apresentado por município, por ser esta uma idade fundamental para comparações e análises, com exceção do Município de Bom Sucesso do Itararé, onde a amostra foi pequena para o município.

A prevalência de cárie dentária nos municípios pesquisados da região de Sorocaba, tendo como referência a escala de severidade da OMS, é baixa (Pinto, 1992). Comparando-se com dados nacionais, em 1986, a prevalência de cárie era muito alta (CPOD de 6,6 aos 12 anos), passando para uma prevalência alta em 1993 (CPOD de 4,8 aos 12 anos) e, em 1996, uma prevalência moderada (CPOD de 3,1 aos 12 anos), sendo de 2,4 no Estado de São Paulo, indicando uma prevalência baixa (Narvai et al., 1999). Todavia, essa prevalência para o Estado de São Paulo não foi confirmada no levantamento epidemiológico realizado pela SES/SP (Narvai \& Castellanos, 1999), pois o CPOD encontrado foi de 3,7 , e, portanto, uma prevalência moderada de cárie. Sendo assim, das 24 regiões do Estado de São Paulo que foram estudadas, apenas $20,8 \%$ apresentaram uma prevalência considerada baixa, e a região de Sorocaba foi uma delas.

Observou-se uma tendência de os municípios de maior porte apresentarem um índice CPOD menor. Isso também foi constatado no levantamento epidemiológico do Estado de São Paulo de 1998 (Narvai \& Castellanos, 1999) e em um amplo estudo realizado no Estado do Paraná no ano de 1996 (Baldani et al., 2002);

Tabela 3

Média de dentes erupcionados, porcentagem de dentes cariados, extraídos e obturados e índice CPOD,

segundo a área de residência e idade. Região de Sorocaba, São Paulo, Brasil, 1998.

\begin{tabular}{|c|c|c|c|c|c|c|c|c|c|c|}
\hline \multirow[t]{3}{*}{ Idade } & \multirow{3}{*}{$\begin{array}{l}\text { Erupcionados } \\
\text { Média }\end{array}$} & \multirow{3}{*}{$\begin{array}{l}\text { CPOD } \\
\text { Média }\end{array}$} & \multirow{3}{*}{$\begin{array}{c}\text { Cariados } \\
(\%)\end{array}$} & \multirow{3}{*}{$\begin{array}{c}\text { Extraídos } \\
(\%)\end{array}$} & \multirow{3}{*}{$\begin{array}{c}\text { Obturados } \\
(\%)\end{array}$} & \multicolumn{4}{|c|}{ CPOD } & \multirow{3}{*}{$\begin{array}{l}\text { Nível de } \\
\text { significância }\end{array}$} \\
\hline & & & & & & \multicolumn{2}{|c|}{ Área com fluoretação } & \multicolumn{2}{|c|}{ Área sem fluoretação } & \\
\hline & & & & & & Média & IC95\% & Média & IC95\% & \\
\hline 5 & 1,7 & 0,1 & 95,5 & 0,0 & 4,6 & $0,1 \mathrm{a}$ & $(0,0-0,1)$ & $0,0 a$ & $(0,0-0,0)$ & $p=0,560$ \\
\hline 6 & 5,6 & 0,1 & 85,5 & 0,0 & 14,6 & $0,1 \mathrm{a}$ & $(0,1-0,1)$ & $0,4 b$ & $(0,1-0,6)$ & $p=0,001$ \\
\hline 7 & 9,4 & 0,5 & 80,8 & 2,9 & 16,3 & $0,5 a$ & $(0,4-0,6)$ & $0,6 a$ & $(0,3-0,8)$ & $p=0,727$ \\
\hline 8 & 12,0 & 0,8 & 71,0 & 1,5 & 27,6 & $0,8 a$ & $(0,7-1,0)$ & $0,8 a$ & $(0,5-1,0)$ & $p=0,588$ \\
\hline 9 & 14,7 & 1,2 & 67,5 & 2,7 & 29,8 & $1,2 \mathrm{a}$ & $(1,0-1,3)$ & $1,3 a$ & $(1,0-1,7)$ & $p=0,342$ \\
\hline 10 & 18,7 & 1,6 & 62,3 & 3,6 & 34,1 & $1,6 \mathrm{a}$ & $(1,5-1,8)$ & $1,5 a$ & $(1,2-1,8)$ & $p=0,609$ \\
\hline 11 & 22,7 & 2,0 & 59,2 & 5,3 & 35,4 & $1,9 a$ & $(1,7-2,1)$ & $2,1 \mathrm{a}$ & $(1,5-2,7)$ & $p=0,638$ \\
\hline 12 & 24,8 & 2,6 & 57,8 & 5,5 & 36,7 & $2,5^{a}$ & $(2,2-2,8)$ & $3,1 \mathrm{a}$ & $(2,4-3,8)$ & $p=0,167$ \\
\hline 5 a 12 & 14,3 & 1,2 & 63,4 & 4,1 & 32,5 & $1,2^{a}$ & $(1,1-1,2)$ & $1,3 a$ & $(1,1-1,5)$ & $p=0,141$ \\
\hline
\end{tabular}

Nota: Letras seguidas de números na horizontal diferem entre si no nível de significância de $5 \%$. 
Figura 1

Porcentagem de crianças de 5 a 12 anos livres de cárie*, considerando a presença ou ausência de flúor nas águas de abastecimento público. Região de Sorocaba, São Paulo, Brasil, 1998.

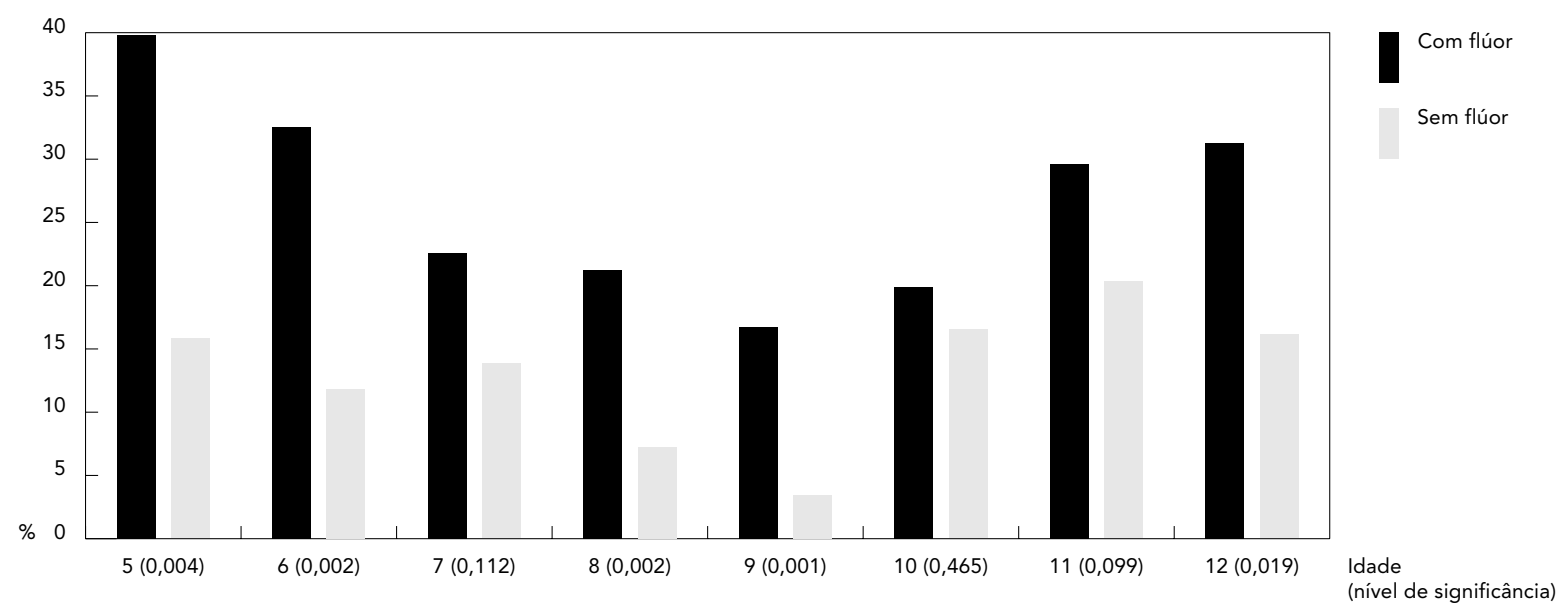

* Consideraram-se como livres de cárie as crianças que apresentaram ceod e CPOD = 0 .

Tabela 4

Prevalência de fluorose dentária em escolares, segundo a idade. Região de Sorocaba, São Paulo, Brasil, 1998.

\begin{tabular}{|c|c|c|c|c|c|c|c|c|c|c|}
\hline \multirow[t]{3}{*}{ Idade } & \multicolumn{4}{|c|}{ Área fluoretada } & \multicolumn{4}{|c|}{ Área não fluoretada } & \multirow{3}{*}{$\begin{array}{c}\text { Total } \\
n\end{array}$} & \multirow{3}{*}{$\begin{array}{l}\text { Nível de } \\
\text { significância }\end{array}$} \\
\hline & \multicolumn{2}{|c|}{ Ausência de fluorose } & \multicolumn{2}{|c|}{ Presença de fluorose } & \multicolumn{2}{|c|}{ Ausência de fluorose } & \multicolumn{2}{|c|}{ Presença de fluorose } & & \\
\hline & $\mathrm{n}$ & $\%$ & $\mathrm{n}$ & $\%$ & $\mathrm{n}$ & $\%$ & $\mathrm{n}$ & $\%$ & & \\
\hline 7 & 363 & 94,0 & 23 & 6,0 & 50 & 76,9 & 15 & 23,1 & 451 & $p<0,001$ \\
\hline 8 & 381 & 92,7 & 30 & 7,3 & 73 & 87,9 & 10 & 12,1 & 494 & $p=0,148$ \\
\hline 9 & 365 & 84,9 & 65 & 15,1 & 70 & 79,5 & 18 & 20,5 & 518 & $p=0,213$ \\
\hline 10 & 363 & 90,1 & 40 & 9,9 & 60 & 70,6 & 25 & 29,4 & 488 & $p<0,001$ \\
\hline 11 & 376 & 85,3 & 65 & 14,5 & 53 & 71,6 & 21 & 28,4 & 515 & $p=0,004$ \\
\hline 12 & 319 & 85,1 & 56 & 14,9 & 42 & 75,0 & 14 & 25,0 & 431 & $p=0,056$ \\
\hline Total & 2.167 & 88,6 & 279 & 11,4 & 348 & 77,2 & 103 & 22,8 & 2.897 & $p<0,001$ \\
\hline
\end{tabular}

contudo, a maioria dos municípios deste encontrava-se nos grupos de alta e moderada prevalência, o que não ocorreu na região de Sorocaba, onde a maioria dos municípios se achava em baixa prevalência de cárie.

A OMS (FDI, 1982) estabeleceu para o ano 2000 algumas metas em saúde bucal. Para a idade de 12 anos, a meta foi alcançada; porém, $30,1 \%$ das crianças ainda apresentam um CPOD maior que 3 , sendo importante que esse grupo receba uma atenção diferenciada e tenha as suas necessidades atendidas. Aos cinco anos, a meta não foi atingida, visto que apenas 37,3\% das crianças estavam livres de cárie, sugerindo que essa população deveria receber maior atenção por parte dos serviços locais, assim como os programas de saúde bucal deveriam ser também direcionados a grupos da população com idade mais precoce.

O índice CPOD, aos 12 anos desta região estudada, foi comparável aos valores relatados por Marthaler et al. (1996), no período de 1991 a 1995, na República Checa, Croácia, Andorra, França, Alemanha, Itália e Eslovênia, de- 
notando-se, assim, um índice favorável de saúde bucal.

Comparando-se também o índice CPOD aos 12 anos de idade com dados de localidades nacionais disponíveis, observou-se que esse valor foi menor que a média do Estado do Paraná em 1996 (Baldani et al., 2002), em Araraquara, São Paulo (Dini et al., 1997), que o CPOD de nove municípios de Goiás no ano de 1994 (Freire et al., 1999), que Teresina (Piauí) em 1992. No entanto, o índice CPOD da região de Sorocaba foi maior que o encontrado nos municípios de Blumenau (Traebert et al., 2001), São Paulo (Narvai et al., 2000), Curitiba, Paulínia e Santos (Basting et al., 1997).

O componente cariado predominou tanto na dentição decídua como na permanente, revelando a necessidade de uma maior cobertura dos serviços odontológicos. Esses valores foram superiores aos valores observados por Narvai et al. (1999) nos levantamentos epidemiológicos nacionais de 1986, 1993 e 1996 e por Traebert et al. (2002) em um município de pequeno porte do Estado de Santa Catarina, em Blumenau (Traebert et al., 2001), na cidade de São Paulo (Narvai et al., 2000) e, ainda, em Piracicaba (Basting et al., 1997). Em Goiás, entretanto, na dentição decídua, a porcentagem de dentes cariados foi maior que a observada no presente estudo, mas na dentição permanente o valor também foi inferior, concordando com os estudos citados anteriormente. Este fato é relevante, pois, apesar de a região de Sorocaba ser considerada uma região com baixa prevalência de cárie, ainda permanece um grupo de crianças necessitando de atenção odontológica integral.

Aos 5 e aos 12 anos de idade, a porcentagem de crianças livres de cárie foi comparativamente maior do que nas idades de 8, 9 e 10 anos, em que as crianças apresentavam dentição mista (Figura 1). Esses dados indicaram uma tendência de diminuição de crianças livres de cárie na fase de dentição mista e, conseqüentemente, uma prevalência de cárie maior, demonstrando que mesmo nesse grupo que recebe atenção odontológica mais direcionada, há períodos de alta prevalência de cárie, ressaltando-se, então, a importância dessas informações, por serem um bom indicador para se verificar a efetividade dos programas de saúde bucal.

Nos municípios com flúor na água de abastecimento público, verificaram-se melhores condições de saúde bucal (Figura 1). Diferentes estudos também verificaram menores índices de cárie em municípios que adicionam flúor na água de abastecimento público (Gill- crist et al., 2001; Jones \& Worthington, 2000; Stephen et al., 2002). Entretanto, neste trabalho, os resultados obtidos no grupo de crianças residentes em municípios sem fluoretação das águas de abastecimento público também se referem a residentes em municípios de pequeno porte, já que na ocasião do sorteio não existiam, na região de Sorocaba, municípios de grande porte sem esse benefício, e o de médio porte (Porto Feliz) foi incluído para as análises, no grupo de municípios com flúor, conforme descrito na metodologia. Dessa forma, devido a esse caráter heterogêneo quanto ao porte dos municípios dessa região do Estado de São Paulo, existem lacunas neste trabalho para responder se o porte do município poderia estar interferindo nos dados relacionados ao papel da fluoretação das águas na região de Sorocaba.

Baldani et al. (2002) encontraram uma diferença significante entre as médias do CPOD dos municípios que apresentavam flúor no sistema de abastecimento público (CPOD médio aos 12 anos de 5,05) em relação aos que não apresentavam (CPOD de 5,95) e uma correlação negativa entre o CPOD e os percentuais de domicílios ligados à rede de água dentre os municípios com flúor, sendo sugerido pelos autores que esses achados tanto podem expressar níveis de cárie menos elevados em melhores condições sócio-econômicas, quanto podem ser um indício de êxito da estratégia de adição de flúor às águas de abastecimento público. No presente estudo, o índice CPOD não apresentou diferenças estatisticamente significativas, mas nos municípios com fluoretação das águas, foi menor a porcentagem de dentes com experiência de cárie $(\mathrm{p}<0,0001)$ e maior porcentagem de dentes hígidos (resultado não apresentado), indicando melhores condições de saúde bucal nesses municípios.

Diferentemente desses achados, Maupomé et al. (2001), ao compararem a prevalência e incidência de cáries em comunidades que fluoretam as águas e as que interromperam a fluoretação, concluem que, em populações de baixa prevalência de cárie, é difícil se detectar diferenças epidemiológicas, devido a diferentes fontes de fluoretos a que esta população está exposta e acesso a serviços.

Interessante observar que houve uma maior prevalência de fluorose nos municípios sem fluoretação das águas de abastecimento público, sendo necessários e importantes futuros estudos para investigar quais as possíveis fontes de exposição a fluoretos a que esta população está sendo submetida.

A fluorose não representou um problema de saúde pública na região de Sorocaba, mas 
essa condição deve ser monitorada permanentemente, dada a importância e benefícios da adição de flúor nos sistemas de abastecimento públicos. Ressalta-se que foram observados alguns casos de fluorose severa, merecendo assim, uma investigação mais detalhada visando identificar as possíveis fontes de exposição a que essas crianças foram submetidas e, caso necessário, intervir de forma a evitar a ocorrência de novos casos na região.

Recomenda-se, por isso, a implementação de estratégias que visem à educação em saúde bucal e priorização da intervenção precoce e efetiva, substituindo, assim, a tendência do acúmulo de necessidades de tratamento em re-

\section{Agradecimentos}

Os autores agradecem a todos que autorizaram que os exames fossem realizados, aos coordenadores de saúde bucal dos municípios, cirurgiões-dentistas e auxiliares que contribuíram para que esta pesquisa fosse realizada. À bibliotecária Marilene Girello pelo auxílio nas correções das referências bibliográficas.

\section{Referências}

BALDANI, M. H.; NARVAI, P. C. \& ANTUNES, J. L. F., 2002. Cárie dentária e condições sócio-econômicas no Estado do Paraná. Cadernos de Saúde Pública, 18:755-763.

BASTING, R. T.; PEREIRA, A. C. \& MENEGHIM, M. C., 1997. Avaliação da prevalência de cárie dentária em escolares do Município de Piracicaba, SP, Brasil, após 25 anos de fluoretação das águas de abastecimento público. Revista Odontológica da Universidade de São Paulo, 11:287-292.

BELTRAN-AGUILAR, E. D.; GRIFFIN S. O. \& LOCKWOOD, S. A., 2002. Prevalence and trends in enamel fluorosis in the United States from the 1930s to the 1980s. Journal of the American Dental Association, 133:157-165.

BRASIL, 1990. Lei n. 8080, de 19 de Setembro de 1990. Dispõe Sobre as Condições para a Promoção, Proteção e Recuperação da Saúde, a Organização e o Funcionamento dos Serviços Correspondentes e Dá Outras Providências. 2 Dezembro $2002<$ http:// portalweb01.saude.gov.br/saude/visao.cfm?id_ area $=169>$.

BRONECKER, M.; MARCENES, W. \& SHEIHAM, A., 2002. Caries reductions between 1995, 1997 and 1999 in preschool children in Diadema, Brazil. In- lação às doenças bucais mais prevalentes, além do monitoramento e avaliação constantes.

\section{Conclusões}

A região de Sorocaba apresenta baixa prevalência de cárie, e a fluorose dentária não foi considerada um problema de saúde pública. As crianças residentes em locais com fluoretação tiveram menor experiência de cárie na dentição decídua e também uma maior porcentagem de crianças apresentaram-se livres de cárie nas idades estudadas, denotando melhores condições de saúde bucal nessas localidades. ternational Journal of Paediatric Dentistry, 12: 183-188.

CHAVES, M. M., 1977. Odontologia Social. 2a Ed. Rio de Janeiro: Editorial Labor do Brasil.

CURY, J. A., 2001. Uso do flúor e controle da cárie como doença. In: Odontologia Restauradora: Fundamentos e Possibilidades (L. N. Baratieri, J. R. S. Monteiro, M. A. C. Andrada, L. C. C. Vieira, A. V. Ritter \& A. C. Cardoso, org.), pp. 32-68, São Paulo: Editora Santos.

DINI, E. L.; FOSCHINI, A. L. R.; BRANDÃO, I. M. G. \& SILVA, S. R. C., 1999. Changes in caries prevalence in 7-12 year-old children from Araraquara, São Paulo, Brazil: 1989-1995. Cadernos de Saúde Publica, 15:617-621.

FDI (Federation Dentaire Internationale), 1982. Global goals for oral health in the year 2000. International Dental Journal, 32:74-77.

FOMON, S. J.; EKSTRAND, J. \& ZIEGLER, E. E., 2000. Fluoride intake and prevalence of dental fluorosis: Trends in fluoride intake with special attention to infants. Journal of Public Health Dentistry, 60:131-139.

FREIRE, M. C. M.; PEREIRA, M. F.; BATISTA, S. M. O.; BORGES, M. R. S.; BARBOSA, M. I. \& ROSA, A. G. 
F., 1999. Prevalência de cárie e necessidades de tratamento em escolares de 6 a 12 anos da rede pública de ensino. Revista de Saúde Pública, 33: 385-390.

FRIAS, A. C., 2000. Estudo de Confiabilidade do Levantamento Epidemiológico de Saúde Bucal - Estado de São Paulo, 1998. Dissertação de Mestrado, São Paulo: Faculdade de Saúde Pública, Universidade de São Paulo.

GILLCRIST, J. A.; BRUMLE, D. E. \& BLACKORFD, J. U., 2001. Community fluoridation status and caries experience in children. Journal of Public Health Dentistry, 61:168-171.

JACKSON, R. D.; KELLY, S. A.; KATS, B.; BRIZENDINE, E. \& STOOKEY, G. K., 1999. Dental fluorosis in children residing in communities with different water fluorides levels: 33-month follow-up. Pediatry Dentistry, 21:248-254.

JONES, C. M. \& WORTHINGTON, H., 2000. Water fluoridation, poverty and tooth decay in 12 year-old children. Journal of Dentistry, 28:389-393.

MARTHALER, T. M.; O'MULLANE, D. M. \& VRBIC, V., 1996. The prevalence of dental caries in Europe 1990-1995. Caries Research, 30:237-255.

MAUPOMÉ, G.; CLARK, D. C.; LEVY, S. M. \& BERKOWITZ, J., 2001. Patterns of dental caries following cessation of water fluoridation. Community Dentistry and Oral Epidemiology, 29:37-47.

NADANOVSKY, P., 2000. O declínio da cárie. In: Saúde Bucal Coletiva (V. G. Pinto, org.), pp. 341-351, São Paulo: Editora Santos.

NADANOVSKY, P. \& SHEIHAM, A., 1995. Relative contribution of dental services to the changes in caries levels of 12-year-old children in 18 industrialized countries in the 1970s and early 1980s. Community Dentistry and Oral Epidemiology, 23:331-339.

NARVAI, P. C. \& CASTELLANOS, R. A., 1999. Levantamento das Condições de Saúde Bucal - Estado de São Paulo, 1998. Relatório de Pesquisa. São Pau- lo: Núcleo de Estudos e Pesquisas de Sistemas de Saúde, Secretaria de Estado de Saúde de São Paulo.

NARVAI, P. C.; CASTELLANOS, R. A. \& FRAZÃO, P., 2000. Prevalência de cárie em dentes permanentes de escolares do Município de São Paulo, SP, 1970-1996. Revista de Saúde Pública, 34:196-200.

NARVAI, P. C.; FRAZÃO, P. \& CASTELLANOS, R. A., 1999. Declínio da experiência de cárie em dentes permanentes de escolares brasileiros no final do século XX. Odontologia e Saúde, 1:25-29.

PINTO, V. G., 1992. Financiamento e organização. In: Saúde Bucal: Odontologia Social e Preventiva (V. G. Pinto, org.), pp. 109-169, São Paulo: Editora Santos.

STEPHEN, K. W.; MacPHERSON, L. M.; GILMOUR, W. H.; STUART, R. A. \& MERRETT, M. C., 2002. A blind caries and fluorosis prevalence study of schoolchildren in naturally fluoridated and nonfluoridated townships of Morayshire, Scotland. Community Dentistry and Oral Epidemiology, 30:7079.

TRAEBERT, J. L.; PERES, M. A.; GALESSO, E. R.; ZABOT, N. E. \& MARCENES, W., 2001. Prevalência e severidade da cárie dentária em escolares de seis a doze anos de idade. Revista de Saúde Pública, 35:283-288.

TRAEBERT, J. L.; SUAREZ, C. S.; ONOFRI, D. A. \& MARCENES, W., 2002. Prevalência e severidade de cárie dentária e necessidade de tratamento odontológico em pequenos municípios brasileiros. Cadernos de Saúde Pública, 18:817-821.

WHO (World Health Organization), 1987. Basics Methods. Geneva: WHO.

WHO (World Health Organization), 1997. Oral Health Surveys, Basics Methods. Geneva: WHO.

Recebido em 14 de agosto de 2002

Versão final reapresentada em 17 de dezembro de 2002 Aprovado em 24 de março de 2003 